\title{
New Inhaler Devices - The Good, the Bad and the Ugly
}

\author{
Federico Lavorini $^{\mathrm{a}} \quad$ Giovanni A. Fontana $^{\mathrm{a}}$ Omar S. Usmani ${ }^{\mathrm{b}}$ \\ ${ }^{a}$ Department of Experimental and Clinical Medicine, Careggi University Hospital, Florence, Italy; \\ ${ }^{b}$ National Heart and Lung Institute, Imperial College London and Royal Brompton Hospital, \\ London, UK
}

\section{Key Words}

Aerosols · Asthma · Chronic obstructive pulmonary

disease $\cdot$ Inhalers $\cdot$ Nebulisers

\begin{abstract}
Drug delivery to the lungs is an effective way of targeting inhaled therapeutic aerosols and treating obstructive airway diseases, such as asthma and chronic obstructive pulmonary disease (COPD). In the past 10 years, several new drugs for the management of asthma and COPD have been marketed and more are under development. These new therapeutic respiratory drugs have been furthered by innovations in all categories of pulmonary drug delivery systems to ensure optimal aerosolisation performance, consistency in efficacy and satisfactory patient adherence. In this review, we discuss the technological advances and innovations in recent inhaler devices and the evolving roles of pressurised metereddose inhalers, dry powder inhalers and nebulisers, as well as their impact on patient adherence to treatment.
\end{abstract}

(c) 2014 S. Karger AG, Basel

\section{Introduction}

The benefits of inhaled therapy for the treatment of obstructive airway diseases, such as asthma and chronic obstructive pulmonary disease (COPD), have been recognised for many years. In comparison with oral or paren- teral formulations, minute but therapeutic drug doses are delivered topically into the airways leading to local efficacy within the lungs [1-3]. Unwanted systemic effects are minimised, as the delivered drug acts with maximum pulmonary specificity combined with a rapid onset and duration of action [1-3]. Consequently, aerosol formulations of bronchodilators and corticosteroids are the mainstay of modern treatment for asthma and COPD $[4,5]$. Aerosols are either solutions containing medications, suspensions of solid drug particles in a gas or dry powder solid particles, which can be generated from devices such as pressurised metered-dose inhalers (pMDIs), dry powder inhalers (DPIs) and nebulisers [1-3]. Inhalers differ in their efficiency of drug delivery to the lower respiratory tract depending on the form of the device, its internal resistance, formulation of medication, particle size, velocity of the produced aerosol plume and ease with which patients can use the device [1-3]. Efficiency of drug delivery may also be influenced by patients' preference, which in turn affects patients' adherence to treatment and, consequently, long-term control of the disease [1].

In recent years, several technical innovations have improved the performance of all existing categories of inhaler devices, and some new delivery systems have been developed that have high delivery efficiencies; notable among these are the so-called 'intelligent inhalers', which allow inhalation to be controlled and patients adherence to treatment to be monitored [6]. Compared with previous devices, the new aerosol drug delivery devices have

\section{KARGER}

E-Mail karger@karger.com

www.karger.com/res
2014 S. Karger AG, Basel

0025-7931/14/0881-0003\$39.50/0
Federico Lavorini, MD, $\mathrm{PhD}$

Department of Experimental and Clinical Medicine Careggi University Hospital, Largo Brambilla 3 IT-50134 Florence (Italy)

E-Mail federico.lavorini@unifi.it 
pulmonary deposition fractions of $40-50 \%$ of the nominal dose, which are significantly higher compared with the low levels of $10-15 \%$ of the nominal dose that were achieved in the past [7]. The increased efficiency of these newer aerosol drug delivery devices means that similar efficacy can be achieved with a lower nominal drug dose [6].

In this article, we review the principal innovative developments in pMDIs, DPIs and nebuliser designs that have recently been introduced or are in the pipeline. One may well wonder what the connection might be between the title of this article and the famous Sergio Leone western movie 'The Good, the Bad and the Ugly'. Well, innovations in existing inhalers, as well as the development of new delivery systems over the last few decades, have led to significant improvements in inhalers efficiency (the good); however, delivery systems are not as harmless as both clinicians and patients may think (the bad), and, more importantly, they may be not as easy to use, thus reducing patients' adherence and consequently treatment efficacy (the ugly). Thus, a thorough understanding of inhaler devices will enable us to limit the 'bad' and the potentially ' 'ugly' and allow the patients the opportunity to derive the 'good' from inhaler devices.

\section{'The Good': Innovations in Pulmonary Drug Delivery Systems}

\section{Pressurised Metered-Dose Inhalers}

The development of the first commercial pMDIs was carried out by Riker Laboratories in 1955 and marketed in 1956 as the first portable, multidose delivery system for bronchodilators. Since that time, the pMDI has become the most widely prescribed inhalation device for drug delivery to the respiratory tract to treat asthma and COPD [8]; between 2002 and 2008, about $48 \%$ of inhaled medications sold in Europe were delivered by pMDIs [8]. The relatively low costs (particularly on a cost-per-dose basis) of pMDIs and the wide variety of medications delivered by pMDIs has contributed to the popularity of this delivery system, particularly in developing countries, and will ensure continued use in developed countries, which are facing increased pressure to reduce health care costs [9]. The pMDI is a portable multidose device that consists of an aluminium canister, lodged in a plastic support, containing a pressurised suspension or solution of micronised drug particles dispersed in propellants. A surfactant (usually sorbitan trioleate or lecithin) is also added to the formulation to reduce particle agglomeration and respon- sible for the characteristic taste of specific inhaler brands. The key component of the pMDI is a metering valve, which delivers an accurately known volume of propellant containing the micronised drug at each valve actuation. The operation principle of the present pMDIs remains similar to the original 1950 push-and-breathe concept: pressing the bottom of the canister into the actuator seating causes decompression of the formulation within the metering valve, resulting in an explosive generation of heterodisperse aerosol droplets that consist of tiny drug particles contained within a shell of propellant. The latter evaporates with time and distance, which reduces the size of the particles that use a propellant under pressure to generate a metered dose of an aerosol through an atomisation nozzle.

Much of the innovation and improvement in pMDI technology has its roots in the significant corporate investment that began in the early 1990s as the industry transitioned to hydrofluoroalkane (HFA) propellant (table 1). Until then, pMDIs used chlorofluorocarbons (CFC) as propellants to deliver drugs; however, in accordance with the Montreal Protocol of 1987, CFC propellants began to be replaced by HFA propellants that do not have ozone-depleting properties [10]. HFA-134a and HFA-227ca are propellants that contain no chlorine and their residence time in the stratosphere is shorter than that of CFCs, and therefore the global warming potential of HFA is substantially lower than that of CFCs. HFA-134a albuterol has been the first HFA-driven pMDI that has received approval in both Europe and the United States. This preparation consists of albuterol suspended in HFA-134a, oleic acid and ethanol; clinical trials have shown this preparation to be bioequivalent to CFC albuterol in both bronchodilator efficacy and side effects [11]. At the present, in most European countries, CFC-driven pMDIs have totally been replaced by HFA inhalers. The components of CFC-driven pMDIs (i.e. canister, metering valve, actuator and propellant) are retained in HFA-driven pMDIs, but their design has been refined. Two approaches were used in the reformulation of HFA-driven pMDIs. The first approach was to show equivalence with the CFC-driven pMDI, which helped regulatory approval, to deliver salbutamol and some corticosteroids. Some HFA formulations were matched with their CFC counterparts on a microgram for microgram basis; therefore, no dosage modification was needed when switching from a CFC to a HFA formulation. The second approach involved extensive changes, particularly for corticosteroid inhalers containing beclomethasone dipropionate, and resulted in 
Table 1. pMDI technology changes

\begin{tabular}{|c|c|c|c|}
\hline & CFC-driven pMDIs & HFA-driven pMDIs & Clinical implications \\
\hline Propellant & CFCs & HFA-134 and HFA-227 & HFAs have no ozone-depleting potential \\
\hline \multirow{3}{*}{$\begin{array}{l}\text { Aerosol } \\
\text { plume }\end{array}$} & High velocity & Reduced velocity & Decreased oropharyngeal deposition \\
\hline & Cold temperature $\left(-29^{\circ} \mathrm{C}\right)$ & Warmer $\left(8^{\circ} \mathrm{C}\right)$ & Reduced chances of 'cold Freon' effect \\
\hline & Spray emitted as a jet & Rounder cloud configuration & Difference in feel and taste \\
\hline \multirow[t]{4}{*}{ Particle size } & MMAD: $3-8 \mu \mathrm{m}$ & MMAD similar to CFCs with suspension pMDIs & No major change \\
\hline & & MMAD: $3-5 \mu \mathrm{m}$ with solution pMDIs & Lower oropharyngeal deposition, enhanced \\
\hline & & MMAD: $1.1-1.3 \mu \mathrm{m}$ with extra-fine solution & deposition in the lung \\
\hline & & pMDIs & Enhanced deposition in peripheral airways \\
\hline \multirow{2}{*}{$\begin{array}{l}\text { Metering } \\
\text { chamber }\end{array}$} & Volume $50-100 \mu \mathrm{l}$ & Smaller chamber & Less chances of leakage during storage \\
\hline & & & $\begin{array}{l}\text { Less chances of loss of prime (i.e. the first actuation } \\
\text { after storage contains a reduced drug dose) }\end{array}$ \\
\hline \multirow[t]{2}{*}{$\begin{array}{l}\text { Actuator } \\
\text { orifice }\end{array}$} & Orifice diameter $\sim 0.5 \mathrm{~mm}$ & Smaller $(\sim 0.2 \mathrm{~mm})$ orifice & $\begin{array}{l}\text { Greater chances of clogging with potential to change } \\
\text { aerosol characteristics }\end{array}$ \\
\hline & & & Reduced spray velocity which facilitates inhalation \\
\hline \multirow[t]{4}{*}{ Formulation } & Creaming of suspension & Ethanol used as solvent & No need to shake the aerosol before use of solution \\
\hline & Variable puff-to-puff & Improved puff-to-puff dosing & pMDIs \\
\hline & dosing 1 & Only a few additional doses provided after a & More consistent clinical efficacy \\
\hline & Tail-off effect & specified number of doses on label claim & $\begin{array}{l}\text { Less chances of misuse because spray content } \\
\text { decreases substantially when additional actuations } \\
\text { are used beyond the specified number of doses on } \\
\text { the label claim }\end{array}$ \\
\hline Priming & Needs priming before use & Variable priming requirements & Check priming instructions according to brand \\
\hline Dose counter & Absent & Present on some devices & Underdosing or overdosing unlikely \\
\hline $\begin{array}{l}\text { Temperature } \\
\text { dependence }\end{array}$ & $\begin{array}{l}\text { Operates best in warm } \\
\text { temperature }\end{array}$ & Less temperature dependence & Losing efficacy unlikely in cold weather \\
\hline Cost & Inexpensive & Expensive & Could change cost-effectiveness \\
\hline
\end{tabular}

solution aerosols with extra-fine particle size (mass median aerodynamic diameter $\sim 1.3 \mu \mathrm{m}$ ) and high lung deposition $[12,13]$; these extensive changes have led to a $2: 1$ dose equivalence ratio in favour of the beclomethasone dipropionate extra-fine HFA-driven pMDI compared to CFC beclomethasone dipropionate $[12,13]$. Patients on regular long-term treatment with a CFC pMDI could safely be switched to a HFA pMDI without any deterioration in pulmonary function, loss of disease control, increased frequency of hospital admissions or other adverse effects [10]. However, when physicians prescribe HFA formulations in place of CFC versions for the first time, they should inform their patients about differences between these products. Compared with CFC-driven pMDIs, many HFA-driven pMDIs have a lower ( 25.5 vs. $95.4 \mathrm{mN}$ ) impact force and a higher $\left(8\right.$ vs. $\left.-29^{\circ} \mathrm{C}\right)$ temperature [14]. These properties partially overcome the 'cold Freon effect' [14] that has caused some patients to stop inhaling their CFC resulting in inconsistent or non-existent dose delivery to the lungs. In addition, compared to CFC pMDIs, most HFA pMDIs have a smaller (from 0.58 to $0.2 \mathrm{~mm}$ ) delivery orifice, which may result in a slower delivery of the aerosol plume, thus facilitating inhalation and producing less mouth irritation [15]. Another difference is that many HFA-driven pMDIs contain co-solvents, such as ethanol. This affects the taste and further increases the temperature and slows the aerosol velocity. pMDIs containing a fixed combination of beclomethasone dipropionate and the long-acting bronchodilator formoterol in a solution formulation with HFA-134a and ethanol with co-solvent $[12,16,17]$ have been developed (Modulite $^{\circledR}$ technology; Chiesi, Parma, Italy). Interestingly, this formulation dispenses an aerosol characterised by extra-fine particles with a lower velocity and at a higher temperature than that obtained when CFCs are used as propellants. These three factors, i.e. smaller particle size, lower plume velocity and less temperature drop, may decrease upper airway impaction and increase airway deposition of particles, particularly to the smaller airways, compared with the same drug administered from a CFC-driven pMDI [16-18].

A frequent complaint from pMDIs users is that it is difficult to determine when their pMDIs will be empty. In a study assessing patients' satisfaction with current pMDIs, $52 \%$ of patients reported that they are extremely 
unsure and $10 \%$ are somewhat unsure of how much medication remains in their current rescue inhaler. With the addition of an integrated dose counter, $97 \%$ of patients reported that they could tell when to replace their inhalers [19]. This has been addressed by the incorporation of dose counters in the pMDI device. The importance of an integrated dose counter in the new pMDIs was emphasised in guidelines issued by the US Food and Drug Administration [20]. GlaxoSmithKline launched the first pMDI with built-in dose counter (Seretide Evohaler ${ }^{\circledR}$ ) in 2004, and dose counters are now incorporated into several new pMDIs. Mechanical dose counters are designed to rely on an active event of firing, such as sound, temperature or pressure change, with their reliability being proven clinically [21]. The primary purpose of dose counters is to inform patients when their inhalers are empty, but dose counters and adherence monitoring devices attached to, or incorporated into, an inhaler could improve adherence to inhaler therapy, especially if the device is coupled to an electronic system reminding patients to take their treatment. Examples of these devices include the DOSER ${ }^{\circledR}$ (Meditrack, South Easton, Mass., USA), Smartinhaler ${ }^{\circledR}$ (Nexus6, Auckland, New Zealand) and the Propeller sensor (Propeller Health, Madison, Wis., USA). These electronic dose counters have relatively high costs and concerns remain regarding the reliability of battery life. Nonetheless, the incorporation of dose counters will become essential for pMDI development to improve disease management by preventing patients from using their inhalers beyond the recommended number of doses and thus receiving suboptimal treatment [22].

One of the biggest challenges associated with effective lung delivery using pMDIs is the difficulty some patients (particularly young children and elderly individuals) have to coordinate device actuation with inspiration [23]; this may lead to a significant reduction in drug deposition in the lungs, and, consequently, less therapeutic effects. Breath-actuated pMDIs are a development from the original press-and-breathe pMDIs to overcome the problem of poor coordination between pMDI actuation and inhalation [1]. Breath-actuated pMDIs contain a conventional pressurised canister and have a flow-triggered system driven by a spring, which releases the dose during inhalation, so that firing and inhaling are automatically coordinated [1]. Newman et al. [24] and Leach et al. [25] observed that drug deposition in the lung of patients using the Autohaler ${ }^{\circledR}$ (3M, St. Paul, Mich., USA), a breath-actuated pMDI, was essentially identical to drug deposition in the lung of patients with good coordination using a press-and-breathe pMDI of the same formulation, but was significantly higher than that for patients with a poor coordination using a press-and-breathe pMDI. Numerous studies have shown improved drug deposition and increased patient confidence that a dose was successfully delivered with the use of breath-actuated pMDI [24, 26, 27]. Using breath-actuated pMDIs, errors are less frequent than with standard pMDIs [28]. Overall, incorporating breath-actuated pMDIs into the patients' regimen may improve overall disease control and reduce health care costs associated with asthma or COPD compared to conventional pMDIs [3] in spite of increased device costs and complexity. The Easi-Breathe ${ }^{\circledR}$ (Teva Pharmaceutical Industries Ltd., New York, N.Y., USA) is similar in function to the Autohaler, but automatically prepares the device for use when the patient opens the mouthpiece cover [26]. When the patient breathes in, the mechanism is triggered and a dose is automatically released into the airstream. The inhaler can be actuated at a very low airflow rate of approximately $20 \mathrm{l} / \mathrm{min}$, which is readily achievable by most patients [26]. Not surprisingly, practice nurses found it easier to teach and patients learned its use easier than with conventional pMDIs [26]. Other breath-actuated pMDIs are the K-Haler ${ }^{\circledR}$ (Clinical Designs, Aldsworth, UK) and the MD Turbo ${ }^{\circledR}$ (Respirics, Raleigh, N.C., USA). With the breath-actuated K-Haler, the drug dose is actuated into a kinked plastic tube, which is straightened by a breath-operated lever, which releases the dose. The MD Turbo was developed as a device designed to fit a variety of commercially available pMDI; it includes an electronic dose counter that shows the patient how much medication is left in the inhaler, and actuation only occurs at a predetermined $(30-60 \mathrm{l} / \mathrm{min})$ inspiratory flow.

Further advances in pMDI technology are represented by devices which incorporate small microprocessors into inhalers themselves; these 'intelligent' inhalers allow inhalation to be controlled and adherence to be monitored [6]. These developments represent significant modifications to the pMDI as a patient interface and clearly require careful analysis of the patient benefits and justification of the additional final unit cost. The SmartMist ${ }^{\circledR}$ (Aradigm Corp., Hayward, Calif., USA) system [6] is a breath-actuated, battery-operated electronic device able to analyse an inspiratory flow profile and automatically actuate the pMDI at a predetermined point in the patient's inhalation when predefined conditions of flow rate and inhaled volume coincide. The SmartMist inhaler effectively guarantees that the patient has good coordination of inhalation and activation of the aerosol jet from the $\mathrm{pMDI}$, and that the inhaled volume and flow rate are 
Table 2. Some current DPIs available on the market for treatment of asthma and COPD

\begin{tabular}{|c|c|c|c|c|}
\hline DPI generation and device & Manufacturer & DPI type & Delivery method & Drugs \\
\hline \multicolumn{5}{|l|}{ First generation } \\
\hline Rotahaler & GlaxoSmithKline & Single dose & Capsule & $\mathrm{SC}$ \\
\hline Aerolizer & Novartis & Single dose & Capsule & Fo, Bud \\
\hline Handihaler & Boehringer & Single dose & Capsule & Tio \\
\hline Breezhaler & Novartis & Single dose & Capsule & Inda, Glico \\
\hline Cyclohaler & Pharmachemie & Single dose & Capsule & Bud, BDP, Sa \\
\hline \multicolumn{5}{|l|}{ Second generation } \\
\hline Turbohaler & AstraZeneca & Multidose & Reservoir & Bud, Fo, Fo+BUD \\
\hline Diskus/Accuhaler & GlaxoSmithKline & Multiunit & Strip package & S, Sa, Flu, Sa+Flu \\
\hline Easyhaler & Orion Pharma & Multidose & Reservoir & $\mathrm{Sa}, \mathrm{BDP}$ \\
\hline Genuair & Almirall & Multidose & Reservoir & $\mathrm{Acl}$ \\
\hline Twisthaler & Schering-Plough & Multidose & Reservoir & Mo \\
\hline Clickhaler & Innovate Biomed & Multidose & Reservoir & $\mathrm{S}, \mathrm{BDP}$ \\
\hline NEXThaler & Chiesi & Multidose & Reservoir & Fo+BDP \\
\hline Ellipta & GlaxoSmithKline & Multiunit & Strip package & Vil + Flu \\
\hline \multicolumn{5}{|l|}{ Third generation } \\
\hline Airmax & Norton Healthcare & Multidose & Reservoir & Fo, Bud \\
\hline
\end{tabular}

$\mathrm{Acl}=$ Aclidinium; $\mathrm{BDP}=$ beclomethasone dipropionate; $\mathrm{Bud}=$ budesonide Fo $=$ formoterol; Flu = fluticasone; Glico = glycopyrronium; Inda = indacaterol; $\mathrm{Mo}=$ mometasone; $\mathrm{S}=$ salbutamol; $\mathrm{Sa}=$ salmeterol; $\mathrm{SC}=$ sodium cromoglicate; Tio = tiotropium; Vil = vilanterol.

both appropriate. A similar technology is used in the AERx Essence ${ }^{\circledR}$ device (Aradigm Corporation), in which a small volume of drug solution is forced through a nozzle array by a breath-actuated piston system [6]. Visual feedback is provided to the patient via a small screen. The device also includes a heater to reduce the droplet size [6].

\section{Dry Powder Inhalers}

DPIs are delivery devices through which a dry powder formulation of an active drug is delivered for local or systemic effects via the pulmonary route [1]. DPIs have a number of advantages over other methods of pulmonary drug delivery, for example, direct delivery of the drug into the deep lungs utilising the patient's respiration, and they are increasingly being explored as devices for the delivery of systemic drugs. Successful delivery of drugs into the deep lungs depends on the interaction between powder formulations and device performance [29]. Dry powders for inhalation are formulated either as loose agglomerates of micronised drug particles with aerodynamic particle sizes $<5 \mu \mathrm{m}$ or as carrier-based interactive mixtures with micronised drug particles adherent to the surface of large lactose carriers [29]. The powder formulation is aerosolised through a DPI device, where the drug particles are separated from the carrier (from drug carrier mixtures) or de-agglomerates drug particles, and the dose is deliv- ered into the patient's deep lungs. In these systems, particle size and flow property, formulation, drug-carrier adhesion, respiratory flow rate and the design of DPI devices significantly affect performance [29]. The physical design of the DPI establishes its specific resistance to airflow (measured as the square root of the pressure drop across the device divided by the flow rate through the device), with current designs having specific resistance values ranging from about 0.02 to $0.2 \mathrm{~cm} \mathrm{H}_{2} \mathrm{O} / 1 / \mathrm{min}$ [30]. To produce a fine powder aerosol with improved delivery to the lung, a low-resistance DPI requires an inspiratory flow of $>90 \mathrm{l} / \mathrm{min}$, a medium-resistance DPI requires 50 $60 \mathrm{l} / \mathrm{min}$, and a high-resistance DPI requires $<50 \mathrm{l} / \mathrm{min}$ [30]. Of note, DPIs with a high resistance tend to produce greater lung deposition than those with a lower resistance [30], but the clinical significance of this is not known.

There is a wide range of DPI devices available on the market (table 2), which deliver either single or multiple doses, and are breath activated or power driven [1, 29]; however, the development of novel devices with new designs continues because the design of a device affects its performance [29]. The challenge is to combine suitable powder formulations with DPI designs that generate small particle aerosols [29].

Based on their design, DPI devices may currently be classified into three broad categories: the first generation, 


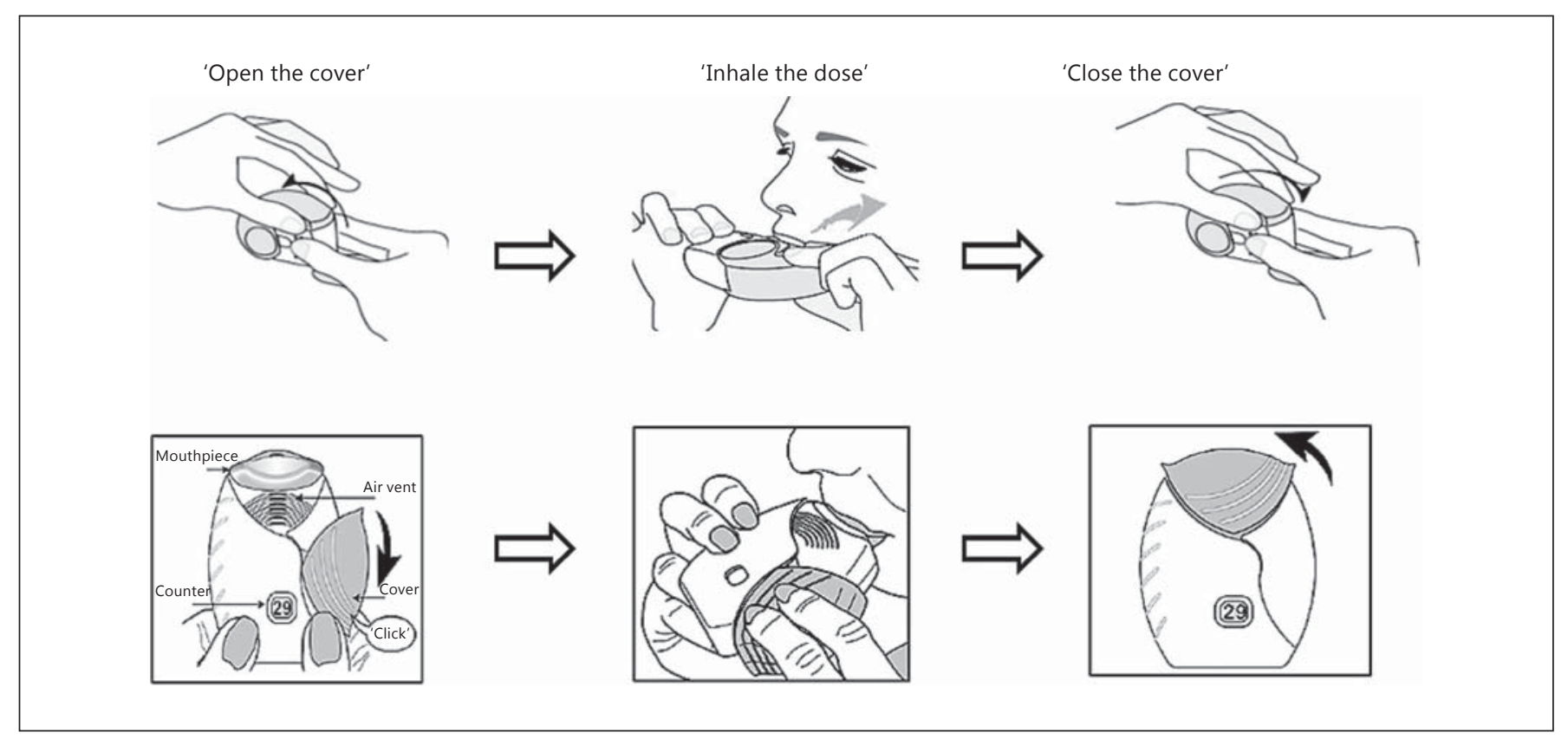

Fig. 1. The three-step operation procedure to use the NEXThaler (upper panels) and the Ellipta (lower panels) inhalers.

single-dose DPIs; the second generation, multiple-dose DPIs, and the third generation DPIs, also known as 'active' or power-assisted DPIs. The first generation, such as for instance the Rotahaler ${ }^{\circledR}$ (GlaxoSmithKline) and the newer Handihaler ${ }^{\circledR}$ (Boehringer Ingelheim, Ingelheim, Germany) and Breezhaler ${ }^{\circledR}$ (Novartis Pharma, Basel, Switzerland), are breath-activated, single-dose devices in which a capsule of powder is perforated in the device with needles fixed to pressure buttons; with these inhalers, drug delivery is affected by particle size and de-agglomeration of drug carrier agglomerates or mixtures delivered by the patient's inspiratory flow. Part of the newly developed DPIs or existing devices used for new powder formulations [31] are still low-resistance capsule-based DPIs. This has the disadvantage that powder properties need to be optimised with respect to both emptying of the capsule and good dispersion. Moreover, the low resistance of the capsule-based DPIs will lead to very high flow rates, which are at the cost of a more central deposition of the drug in the lung [29]. The second-generation DPIs fall into two main categories: multidose DPI devices, i.e. they measure the dose themselves from a powder reservoir, or multi-unit DPI devices, i.e. they dispense individual doses which are pre-metered into blisters, disks, dimples, tubes and strips by the manufacturer [32-34]. The Turbuhaler ${ }^{\circledR}$ (AstraZeneca, Södertälje, Sweden) and Diskus ${ }^{\circledR}$ (GlaxoSmithKline) are representatives of the former and latter categories, respectively, although many other different designs are presently in development. All these DPIs have some essential components incorporated in the device, such as a drug holder, air inlet, de-agglomeration compartment and a mouthpiece. The design of DPIs is developed in such a way that the device should induce sufficient turbulence and particle-particle collisions to detached drug particles from the carrier surface (interactive mixtures) or de-agglomerates particles from large agglomerates of drugs only. Drug delivery to the lungs with these inhalers ranges between 12 and $40 \%$ of the emitted dose [32-34]. The more recently developed second-generation DPIs which are commercially available are the NEXThaler ${ }^{\circledR}$ (Chiesi), Ellipta ${ }^{\circledR}$ (GlaxoSmithKline) and the Genuair ${ }^{\circledR}$ (Almirall S.A., Barcelona, Spain). The NEXThaler delivers the fixed dose combination of formoterol fumarate and beclomethasone dipropionate as extra-fine particles for asthma treatment while the Ellipta device has been developed to deliver the new combination of the inhaled corticosteroid fluticasone furoate combined with the new long-acting $\beta$-adrenergic bronchodilator vilanterol as a once-daily inhaled maintenance therapy for asthma and COPD. Both of these devices are multidose DPIs with a simple three-step operation procedure, which can take into account typical human behaviour [35]: open the cover, inhale from the mouthpiece and close the cover (fig. 1). The NEXThaler 


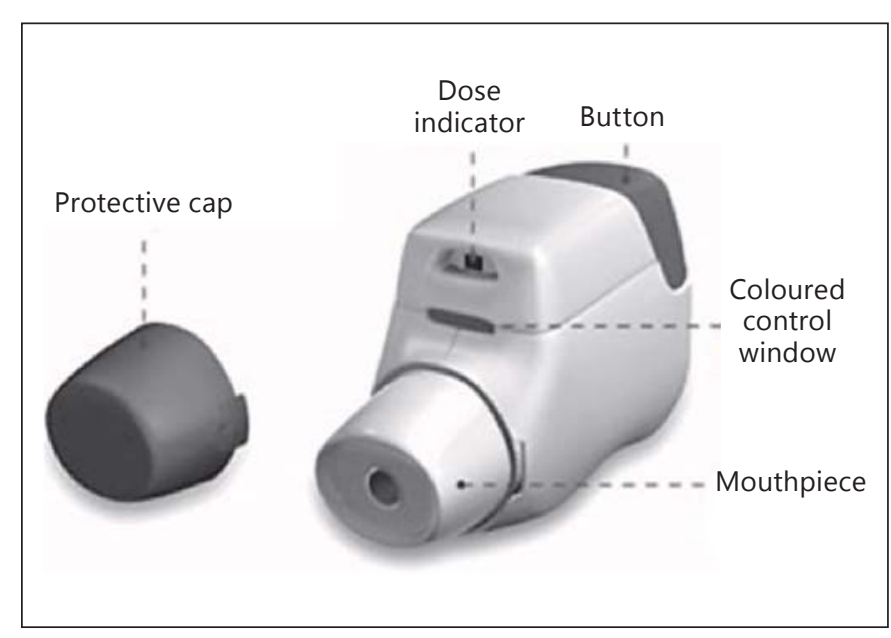

Fig. 2. General design and features of the Genuair inhaler.

is equipped with an innovative full-dose feedback system incorporating a novel breath-actuated mechanism guaranteeing that the dose is released only when a threshold inspiratory flow of $35 \mathrm{l} / \mathrm{min}$ is achieved. A dose protector covers the dose and prevents the dose from being inhaled until the mechanism is triggered by a flow rate that allows complete de-aggregation and delivery of the full dose [36]. Of note, the NEXThaler is the only DPI delivering extra-fine particles and this unique characteristic depends on specific physicochemical properties of the powder formulation, as well as on the innovative de-aggregation release system [36]. The Ellipta is a multi-unit DPI which includes a dose counter; a recent exploratory study has shown that several attributes of the Ellipta, such as ease of use and simplicity of operation, the visibility and ease of interpretation of the dose counter, the feel and fit of the inhalation mouthpiece, and design ergonomics, are viewed positively by asthma and COPD patients [37]. Noticeably, the Ellipta was preferred to other inhalers by interview participants with asthma and COPD [37]. The Genuair (fig. 2) is a novel multidose DPI designed to deliver the long-acting anti-muscarinc bronchodilator aclidinium bromide from a non-removable cartridge [38]. The inhaler design includes visual and acoustic feedback to reassure patients that they have taken their medication correctly, a dose indicator and a lockout mechanism to prevent the use of an empty inhaler. The inhaler has medium airflow resistance and uses an optimised dispersion system to ensure effective de-agglomeration of the inhalation powder [38]. In vitro studies have demonstrated that the inhaler delivers a reproducible aerodynamic aerosol quality and is reliable under various thermal and mechanical stress conditions [38]. Further studies in vitro have demonstrated that the total emitted dose and fine particle dose are both consistent over a range of inhalation flows from 45 to $95 \mathrm{l} / \mathrm{min}$, as well as being independent of inhalation volume (2 vs. 4 litres) and storage conditions [38]. In healthy subjects, delivery of $200 \mu \mathrm{g}$ of aclidinium bromide via the inhaler achieved high lung deposition (approximately $30 \%$ of the metered dose) [39]. The high lung deposition observed in this study is consistent with the high fine particle dose generated from the inhaler in vitro [38]. A further study has shown that patients with moderate or severe COPD can generate sufficient inspiratory airflow through the inhaler to reliably inhale the full dose and reset the inhaler [40]. The third and newer generation DPIs are 'active', power-assisted devices, which incorporate battery-driven impellers and vibrating piezo-electric crystals (e. g. MicroDose ${ }^{\circledR}$; MicroDose Therapeutx, Monmouth Junction, N.J., USA), to disperse drug from the formulation, thus reducing the need for the patient to generate a high inspiratory flow rate, an advantage particularly for patients with impaired lung function [33, 41]. Due to the presence of an energy source, active DPI devices enable respiratory force-independent dosing precision and reproducible aerosol production. In vitro studies have shown that active DPIs are able to produce aerosols characterised by fine particle fraction values in the range of $50-70 \%$ [41]. These devices are obviously more sophisticated than passive DPIs, and they are likely to be relatively expensive devices for asthma and COPD therapy, but could play a future role in the delivery of other drugs, such as peptides or proteins. The development of novel electronic DPIs, such as the MicroDose device, has shown that features such as dose delivery confirmation, adherence monitoring and dosing reminders can be incorporated into portable inhalers at relatively low cost [6].

\section{Nebulisers}

Various types of nebulisers are available on the market, and several studies have indicated that performance varies between manufacturers and also between nebulisers from the same manufacturers [42-44]. Jet and ultrasonic nebulisers have recently been joined by a third type using a vibrating membrane or mesh [42-44]. The jet (or pneumatic) nebulisers (e. g. LC Sprint ${ }^{\circledR}$, PARI GmbH, Starnberg, Germany) remain the most commonly used nebulisers in clinical practice; they generate aerosol particles as a result of the impact between a liquid and a jet of high velocity gas (usually air or oxygen) in the nebuliser chamber. A 6-8 1/min flow and a fill volume of $4-5$ 
$\mathrm{ml}$ are generally recommended, unless some nebulisers are specifically designed for a different flow and a smaller or larger fill volume [45]. With jet nebulisers, treatment times are generally long, the air compressors are heavy and noisy, and mechanical shear forces can affect certain medications. The longer nebulisation time with a greater fill volume can be reduced by increasing the flow used to power the nebuliser; however, increasing the flow decreases the droplet size produced by the nebuliser. Dead volume is the volume that is trapped inside the nebuliser and typically it is $0.5-1 \mathrm{ml}$. Because of the evaporative loss within the nebuliser, the solution becomes increasingly concentrated and cools during nebulisation.

Ultrasonic nebulisers (e.g. PolyGreen KN-9210; PolyGreen, Stahnsdorf, Germany) use a rapidly (>1 MHz) vibrating piezo-electric crystal to produce aerosol particles [42-44]. Ultrasonic vibrations from the crystal are transmitted to the surface of the drug solution where standing waves are formed. Droplets break free from the crest of these waves and are released as aerosol. The size of droplets produced by the ultrasonic nebuliser is related to the frequency of oscillation [42-44]. Although ultrasonic nebulisers operate silently, and can nebulise solutions more quickly than jet nebulisers, they are not suitable for suspensions and their piezo-electric crystal can heat the liquid drug in the reservoir, which renders it inappropriate for thermal-labile medications [3].

Vibrating mesh nebulisers are the newest technologies which overcome the disadvantages of both jet and ultrasonic nebulisers [46-48]. These new-generation nebulisers are either active or passive systems. In active devices (e.g. eFlow $^{\circledR}$, PARI GmbH), the aperture plate vibrates at a high frequency and draws the solution through the apertures in the plate. In passively vibrating mesh devices (e.g. MicroAir ${ }^{\circledR}$, Omron Healthcare, Hoofddorp, The Netherlands), the mesh is attached to a transducer horn and vibrations of the piezo-electric crystal that are transmitted via the transducer horn force the solution through the mesh to create an aerosol. The PARI eFlow is designed to be used with either a very low residual volume to reduce drug waste or with a relatively large residual volume, so that it can be used instead of conventional jet nebulisers with the same fill volume [48]. Vibrating mesh nebulisers have a number of advantages over other nebuliser systems: they have greater efficiency, precision and consistency of drug delivery, are quiet and generally portable $[46,47]$. However, they are also significantly more expensive than other types of nebuliser and require a significant amount of maintenance and cleaning after each use to prevent the build-up of deposit and blockage of the aper- tures, especially when suspensions are aerosolised, and to prevent colonisation by pathogens [46].

The principle of all the above-mentioned nebuliser types is that aerosol is generated continuously throughout the patient's entire respiratory cycle (fig. 3). Thus, a large proportion of medication is lost during exhalation, resulting in inefficient aerosol drug delivery and variable dosing. Significant enhancements in drug delivery by nebulisers are possible by coordinating nebulisation with inspiration, i.e. the nebuliser is turned off during expiration ('breath-actuated' nebulisers; fig. 3) or utilising the patient's inspiratory flow through the nebuliser to increase drug delivery ('breath-enhanced' nebulisers; fig. 3) $[42,43]$. Both types of nebulisers are modifications of the 'conventional' jet nebulisers specifically designed to improve their efficiency by increasing the amount of aerosol delivered to the patient with less wastage of aerosol during exhalation $[42,43]$. The breath-enhanced jet nebuliser (e.g. LC ${ }^{\circledR}$ Plus; PARI GmbH) uses two one-way valves to prevent the loss of aerosol to the environment. When the patient inhales, the inspiratory valve opens and aerosol vents through the nebuliser; exhaled aerosol passes through an expiratory valve in the mouthpiece. Breathactuated jet nebuliser are designed to increase aerosol delivery to the patient by means of a breath-actuated valve (e.g AeroEclipse ${ }^{\circledR}$; Monoghan Medical Corporation, Plattsburgh, N.Y., USA) that triggers aerosol generation only during inspiration. Both the breath-enhanced and breath-actuated nebulisers increase the amount of inspired aerosol with shorter nebulisation time than 'conventional' jet nebulisers [42]. More recently, much greater control of nebulised aerosol delivery has been afforded by the coupling of software control with nebulisers [4951]. These new-generation, 'adaptive aerosol delivery' nebulisers monitor the patient's breathing pattern and continuously adjust the delivery of nebulised medication accordingly, thus leading to accurate high-dose pulmonary drug deposition in a much shorter time. By monitoring pressure changes relative to flow over the first three breaths, these delivery systems establish the shape of the breathing pattern and then use this to provide a timed pulse of aerosol during the first $50 \%$ of each tidal inspiration. Monitoring of the breathing pattern continues throughout the delivery period and any change in the breathing pattern is taken into account during the remainder of the delivery period. Furthermore, if no inhalation is registered, the system will cease delivery until the patient recommences breathing on the system [49-51]. Since the pulsed dose is only provided in the first $50 \%$ of each breath, and the software can calculate the amount of 


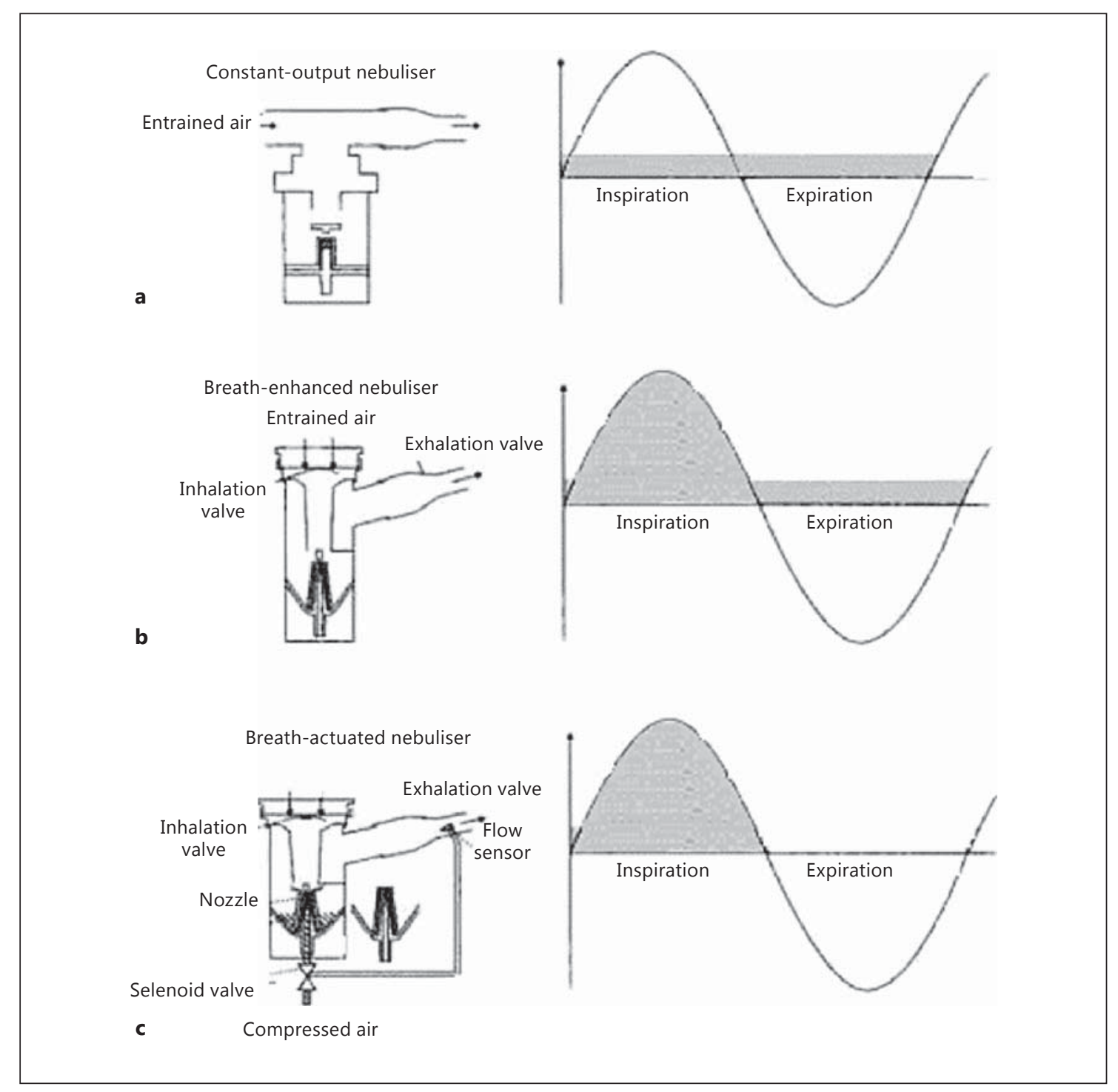

Fig. 3. Differences in the jet nebuliser design and aerosol output are indicated by the shaded area. a Constantoutput pneumatic jet nebulizer. b Breath-enhanced jet nebuliser. c Breath-actuated jet nebuliser.

drug given per pulse, the precise dose of drug can be delivered before the system stops [49-51]. The I-neb ${ }^{\circledR}($ Philips Respironics Healthcare, Chichester, UK) and the Prodose $^{\circledR}$ (Profile Therapeutics, Bognor Regis, UK) are examples of commercially available adaptive aerosol delivery systems approved in the US for delivery of inhaled prostacyclin to patients with pulmonary arterial hypertension and in Europe as multipurpose nebulisers. Both of these nubulisers use an adaptive aerosol delivery disk containing a microchip and antenna to control drug delivery. The I-neb is a vibrating mesh nebuliser, whereas the Prodose is powered by a compressor. In addition to delivering a precise drug dose, other useful features of the I-neb are the provision of feedback to the patient on dose completion along with details on each treatment. These data can be transmitted via a modem to a remote location, which enables continuing assessment of adherence of the patient to the drug regimen [3].

The AKITA $^{\circledR}$ system (Vectura, Chippenham, UK) contains a SmartCard electronic control unit with an air compressor, which is coupled to either jet or vibrating mesh nebulisers [52]. The SmartCard software operates the air compressor unit to regulate the patient's inhalation such that the AKITA system can accurately control 
dose delivery and target nebulised aerosol to specific regions of the lungs. A vibrating mesh nebuliser using the AKITA system deposits $70 \%$ of the nebuliser fill in the lungs of patients with $\alpha_{1}$-antitrypsin deficiency [53]. Two different nebulisers controlled by AKITA were shown to increase total and peripheral lung deposition of an $\alpha_{1^{-}}$ protease inhibitor in patients with COPD compared to two other nebulisers used with spontaneous breathing [54]. In an open-label, pilot trial [55], budesonide was administered by jet nebulisation with or without control by the AKITA system to children with asthma. Compared to regular jet nebulisers, the AKITA system achieved similar or better efficacy, and was well accepted by children and their parents. It also reduced the time for inhalation as well as the required nebulised doses [55]. The significance of these results is reflected in a study by Hofmann [56] who found the AKITA system to be an excellent driver of patient adherence, achieving an exceptional $92 \%$ adherence rate in children. This also highlighted the usefulness of the logging software of the system for checking patient adherence by doctors and for clinical trials [56]. Beyond adherence, clinical efficacy could also be improved by controlling specific regional deposition. Targeting of small airways in asthma by inhaled medications can be challenging. Therefore, there may be an opportunity to reduce side effects associated with systemic steroid uptake in patients with severe asthma who are not sufficiently controlled using regular inhalation, and systemic steroids are often indicated and associated with side effects. By programming the AKITA system to target the peripheral airways, Janssens and Overweel [57] found that systemic steroid exposure in children with severe asthma was reduced as were hospital admissions.

\section{Other Inhaler Technology}

Portable inhaler technology using principles other than those used in pMDIs and DPIs are now entering the market, and are designed with patient ease of use in mind. The development of soft mist inhalers does fall within the definition of a nebuliser, as they transform aqueous liquid solution to liquid aerosol droplets suitable for inhalation. However, at variance with the traditional nebuliser designs, they are hand-held multidose devices that have the potential to compete with both pMDIs and DPIs on the portable inhaler market. At the present, the only soft mist inhaler currently marketed in some European countries is the Respimat ${ }^{\circledR}$ inhaler (Boehringer Ingelheim). This device does not require propellants since it is powered by the energy of a compressed spring inside the inhaler. Individual doses are delivered via a precisely engineered noz- zle system as a slow-moving aerosol cloud (hence the term 'soft mist') [58]. Scintigraphic studies have shown that, compared to a CFC-based pMDI, lung deposition is higher (up to 50\%) and oropharyngeal deposition is lower [58]. Respimat is a 'press-and-breathe' device, and the correct inhalation technique closely resembles that used with a pMDI. However, although coordination between firing and inhaling is required, the aerosol emitted from Respimat is released very slowly, with a velocity of approximately four times less than that observed with a CFC-driven pMDI [58]. This greatly reduces the potential for drug impaction in the oropharynx. In addition, the relatively long duration over which the dose is expelled from the Respimat (about $1.2 \mathrm{~s}$ compared with $0.1 \mathrm{~s}$ from traditional pMDIs) would be expected to greatly reduce the need to coordinate actuation and inspiration, thus improving the potential for greater lung deposition. Although the Respimat has been used relatively little in clinical practice to date, clinical trials seem to confirm that drugs delivered by the Respimat are effective at correspondingly smaller doses in patients with obstructive airway disease [59].

\section{The 'Bad' and the 'Ugly': Poor Inhaler Technique and Its Consequences}

A fundamental requirement that underlies all inhaled therapies is the need to use the inhaler correctly in order to achieve the optimal therapeutic response from the drug. Published evidence shows that, when used correctly, there is little difference in clinical efficacy between different inhaler types [2]. Despite the development of several new and improved types of inhaler devices, there has been no sustained improvement over the past 35 years in patients' ability to use their inhalers. In fact, several studies have reported that up to $50-60 \%$ of patients with asthma or COPD cannot use their inhalers (either pMDIs or DPIs) well enough to benefit from the treatment $[23,60]$. These numbers are even more depressing considering that between 40 and $85 \%$ of health care professionals, who should readily be able to teach patients how to use their inhalers correctly, do not seem to be able to perform that task properly - and doctors are the worst amongst all health care professionals [61].

Poor inhaler technique has clinical consequences, which have been documented for asthma patients taking inhaled corticosteroids delivered by pMDIs: instability of asthma was more frequent in patients with a poor inhaler technique than in those with a good technique [62]. In a large cross-sectional study involving over 1,600 asthma
Lavorini/Fontana/Usmani 
outpatients, the finding of just one critical error in the inhalation technique, irrespective of the inhalation device (DPI or pMDI), was associated with increased emergency room visits, hospitalisation and oral medication prescription [63]. More recently, Levy et al. [64] retrospectively evaluated pMDI use in patients with mild-to-moderate asthma and correlated the patients' inhaler technique with the level of asthma control. Noticeably, the patients' pMDI inhaler technique was objectively evaluated by using the Vitalograph Aerosol Inhalation Monitor [65], a training device aimed at assessing three crucial steps needed for correct pMDI usage: slow ( $<50 \mathrm{l} / \mathrm{min})$ inhalation flow; synchronisation between inhaler actuation and inhalation, and a 5-second breath hold pause following inhalation. The authors observed that patients who displayed significant errors when using pMDIs had higher risks of poor asthma control and more bursts of systemic corticosteroid prescriptions than those who operated pMDIs correctly [64]. Of note, patients who were using breath-actuated inhalers had better asthma control than those using pMDIs alone. Synchronisation, i.e. achieving the correct inhalation flow following actuation, was the main step in the inhalation technique which most patients failed [64]. The results of this study confirm the relationship between inhaler misuse and poor asthma control, and reinforce the notion of the importance of patient training for efficient drug inhalation. Patients' ability to handle inhalers correctly is a crucial issue for the choice of the most appropriate inhaler device for a given patient [1]. Adherence to therapy is likely to be influenced by patients' attitudes and their experience in using the device, and if the patient feels that their treatment is not working, adherence is likely to be poor, resulting in reduced efficacy of treatment [66]. Evidence shows that patients' competence in the self-administration of inhaled medications is improved by educational interventions [64], and repeated training in correct inhaler use improves asthma symptoms, quality of life and lung function, and reduces the use of relief medications as well as emergency hospital admissions $[66,67]$.

A poor inhaler technique also has financial consequences, with one review [67] estimating that about a quarter of all expenses on inhalers is wasted owing to a poor inhaler technique.

\section{Future Directions and Conclusions}

In the past $10-15$ years, several innovative developments have advanced the field of inhaler design. However, there has been little effort made in that time to systemati- cally bring the medical community up to speed, with virtually no clinician training to ensure understanding of how these devices work, no less how to choose the best device to meet the needs of a specific patient. Although many inhalers incorporate features providing efficient aerosol delivery for treatment of asthma and COPD, there is no perfect inhaler, and each has advantages and disadvantages [1], but there is increasing recognition that a successful clinical outcome is determined as much by the choice of the appropriate inhaler device as by the drugs that go in them. Researchers reported that up to $60 \%$ of patients do not use their inhaler well enough to benefit from their prescribed medication $[23,60]$, which correlates to the number of practitioners who are unable to properly use and teach the use of these devices to their patients [61]. This situation results in financial resources wasted not only in drugs that are ineffective but also in the acute and critical care of patients. Health care costs for patients prescribed inhaled drugs continue to increase while many do not benefit from the prescribed medication. It is less an issue of the medications not being effective when properly administered, but more the failure to properly administer those medications. However, as inhalation is likely to remain the delivery route of choice for the foreseeable future, there is a need to develop inhaler devices which are easy to use and deliver a consistent dose of drug to the lungs, which may improve patient compliance with treatment and eventually lead to better asthma control and COPD outcome. The recent advances in aerosol delivery systems and formulations indicate certain trends in the field. Breath-actuated inhalers and inclusion of dose counters are anticipated to improve asthma control; nebulisers, particularly software-aided systems which can accurately direct lung deposition and total dosing, will play a crucial role in improving the reliability of clinical trials on inhaled therapeutics. Ensuring effective inhalation therapy depends upon many factors that are related to the patient, device, drug and environment. Understanding the characteristics of the aerosol device, as well as the knowledge, attitudes and preference of patients will influence satisfaction with aerosol therapy and optimise clinical outcomes. Therefore, the clinician's familiarity with inhalers and their ability to understand their patients' needs and preferences are important for the selection of the best aerosol device for their patients. While there will be new devices and drug device combinations introduced over the next 5 years, little will change until we actively educate health care professionals on how to select the best available device to meet each patient's needs, modify that selection as the abilities, needs or preferences of the pa- 
tient changes, and commit resources for assuring that patients and caregivers are trained to properly use and maintain their devices. Only through recognition of the 'good' inhaler we will avoid the 'bad' and the 'ugly'.

\section{Acknowledgements}

O.S. Usmani is a recipient of a UK National Institute for Health Research Career Development Fellowship and is supported by the Respiratory Disease Biomedical Research Unit at the Royal Brompton and Harefield NHS Foundation Trust and Imperial College London.

\section{Financial Disclosure and Conflicts of Interest}

In the past 5 years, F.L. received lecture fees or reimbursements for attending meetings from AstraZeneca, Chiesi, MedaPharma, Mundipharma, Menarini and Teva. In the past 5 years, G.A.F. received lecture fees for attending meetings and grants from $\mathrm{Me}$ narini, Mundipharma, Edmond Pharma and Dompé. F.L. is a member of the Aerosol Drug Management Improvement Team, a non-commercial consortium of European physicians with special interest in studying and promoting the correct use of therapeutic aerosols. In the past 5 years, O.S.U. received lecture fees or reimbursements for attending meetings from Chiesi, GlaxoSmithKline and Mundipharma.

\section{References}

1 Laube BL, Janssens HM, de Jongh FHC, et al: What the pulmonary specialist should know about the new inhalation therapies. Eur Respir J 2011;37:1308-1331.

2 Dolovich MB, Ahrens Rc, Hess DR, et al: Device selection and outcomes of aerosol therapy: evidence-based guidelines: American College of Chest Physicians/American College of Asthma, Allergy, and Immunology. Chest 2005; 127:335-371.

-3 Dolovich MB, Dhand R: Aerosol drug delivery: developments in device design and clinical use. Lancet 2011:377:1032-1045.

4 Global Initiative for Asthma: Global Strategy for Asthma Management and Prevention. http://www.ginaasthma.com (updated December 2012).

5 Global Initiative for Chronic Obstructive Lung Disease: Global Strategy for Diagnosis, Management, and Prevention of COPD. http://www.goldcopd.org/uploads/users/ files/GOLD_Report_2011_Jan21.pdf (2011 update)

6 Newman S: Improving inhaler technique, adherence to therapy and the precision of dosing: major challenges for pulmonary drug delivery. Expert Opin Drug Deliv 2014;11:365378.

7 Heyder J: Deposition of inhaled particles in the human respiratory tract and consequences for regional targeting in respiratory drug delivery. Proc Am Thorac Soc 2004;1:315320.

8 Lavorini F, Corrigan CJ, Barnes PJ, et al: Retail sales of inhalation devices in European countries: so much for a global policy. Respir Med 2011;105:1099-1113.

-9 Doan Q, Shefrin A, Johnson D: Cost-effectiveness of metered-dose inhalers for asthma exacerbations in the pediatric emergency department. Pediatrics 2011;127:1105-1111. Hendeles L, Colice GL, Meyer RJ: Withdraw-
al of albuterol inhalers containing chlorofluorocarbon propellants. N Engl J Med 2007;356: 1344-1351.
11 Ross DL, Gabrio BJ: Advances in metered dose inhaler technology with the development of a chlorofluorocarbon-free drug delivery system. J Aerosol Med 1999;12:151-160.

12 Ganderton D, Lewis D, Davies R, et al: Modulite: a means of designing the aerosols generated by pressurized metered dose inhalers. Respir Med 2002;96(suppl D):S3-S8.

13 Leach CL: The CFC to HFA transition and its impact on pulmonary drug development. Respir Care 2005;50:1201-1208.

14 Sanchis J, Corrigan C, Levy ML, Viejo JL: Inhaler devices - from theory to practice. Respir Med 2013;107:495-502.

15 Gabrio BJ, Stein SW, Velasquez DJ: A new method to evaluate plume characteristics of hydrofluoroalkane and chlorofluorocarbon metered dose inhalers. Int J Pharm 1999;186: 3-12.

16 Acerbi D, Brambilla G, Kottakis I: Advances in asthma and COPD management: delivering CFC-free inhaled therapy using Modulite technology. Pulm Pharmacol Ther 2007;20: 290-303.

17 Dhillon S, Keating GM: Beclometasone dipropionate/formoterol: in an HFA-propelled pressurised metered-dose inhaler. Drugs 2006;66:1475-1483.

18 Usmani OS, Biddiscombe MF, Barnes PJ: Regional lung deposition and bronchodilator response as a function of beta2-agonist particle size. Am J Respir Crit Care Med 2005;172: 1497-504.

19 Given J, Taveras H, Iverson H, Lepore M: Prospective, open-label assessment of albuterol sulfate hydrofluoroalkane metered-dose inhaler with new integrated dose counter. Allergy Asthma Proc 2013;34:42-51.

20 FDA: Guidance for Industry: Integration of Dose-Counting Mechanisms into MDI Drug Products. Rockville, FDA, 2003.

21 Weinstein C, Staudinger H, Scott I, Amar NJ, LaForce C: Dose counter performance of mometasone furoate/formoterol inhalers in subjects with asthma or COPD. Respir Med 2011; 105:979-988.
22 Conner JB, Buck PO: Improving asthma management: the case for mandatory inclusion of dose counters on all rescue bronchodilators. J Asthma 2013;50:658-563.

23 Crompton GK, Barnes PJ, Broeder M, et al: The need to improve inhalation technique in Europe: a report from the Aerosol Drug Management Improvement Team. Respir Med 2006;100:1479-1494.

24 Newman SP, Weisz A, Talaee N, Clarke S: Improvement of drug delivery with a breath actuated pressurised aerosol for patients with poor inhaler technique. Thorax 1991;46:712716.

25 Leach CL, Davidson PJ, Hasselquist BE, Boudreau RJ: Influence of particle size and patient dosing technique on lung deposition of HFAbeclomethasone from a metered dose inhaler. J Aerosol Med 2005;18:379-385.

26 Price DB, Pearce L, Powell SR, Shirley J, Sayers MK: Handling and acceptability of the EasiBreathe device compared with a conventional metered dose inhaler by patients and practice nurses. Int J Clin Pract 1999;53:31-36.

27 Hampson NB, Mueller MP: Reduction in patient timing errors using a breath-activated metered dose inhaler. Chest 1994;106:462465 .

28 Lenny J, Innes J, Crompton GK: Inappropriate inhaler use: assessment of use and patient preference of seven inhalation devices. Respir Med 2000;94:496-500.

29 Hoppentocht M, Hagedoorn P, Frijlink HW, de Boer AH: Technological and practical challenges of dry powder inhalers and formulations. Adv Drug Deliv Rev 2014 DOI: 10.1016/j.addr.2014.04.004.

30 Azouz W, Chrystyn H: Clarifying the dilemmas about inhalation techniques for dry powder inhalers: integrating science with clinical practice. Prim Care Respir J 2012;21:208-213.

31 Geller DE, Weers J, Heuerding S: Development of an inhaled dry-powder formulation of tobramycin using PulmoSphere ${ }^{\mathrm{TM}}$ technology. J Aerosol Med Pulm Drug Deliv 2011;24: 175-182. 
-32 Newman SP, Busse WW: Evolution of dry powder inhaler design, formulation, and performance. Respir Med 2002;96:293-304.

- 33 Islam N, Gladki E: Dry powder inhalers (DPIs) - a review of device reliability and innovation. Int J Pharm 2008;360:1-11.

-34 Atkins PJ: Dry powder inhalers: an overview. Respir Care 2005;50:1304-1312.

-35 Smith IJ, Bell J, Bowman N, Everard M, Stein S, Weers JG: Inhaler devices: what remains to be done? J Aerosol Med Pulm Drug Deliv 2010;23(suppl 2):S25-S37.

-36 Voshaar T, Spinola M, Linnane P, Campanini A, Lock D, Lafratta A, Scuri M, Ronca B, Melani AS: Comparing usability of NEXThaler with other inhaled corticosteroid/long-acting $\beta_{2}$-agonist fixed combination dry powder inhalers in asthma patients. J Aerosol Med Pulm Drug Deliv 2013, Epub ahead of print.

- 37 Svedsater H, Dale P, Garrill K, Walker R, Woepse MW: Qualitative assessment of attributes and ease of use of the ELLIPTA ${ }^{\mathrm{TM}}$ dry powder inhaler for delivery of maintenance therapy for asthma and COPD. BMC Pulm Med 2013;13:72.

- 38 Chrystyn H, Niederlaender C: The Genuair inhaler: a novel, multidose dry powder inhaler. Int J Clin Pract 2012;66:309-317.

- 39 Newman SP, Sutton DJ, Segarra R, Lamarca R, de Miquel G: Lung deposition of aclidinium bromide from Genuair, a multidose dry powder inhaler. Respiration 2009;78:322328.

40 Magnussen H, Watz H, Zimmermann I, et al: Peak inspiratory flow through the Genuair inhaler in patients with moderate or severe COPD. Respir Med 2009;103:1832-1837.

41 Islam N, Cleary MJ: Developing an efficient and reliable dry powder inhaler for pulmonary drug delivery - a review for multidisciplinary researchers. Med Eng Phys 2012;34: 409-427.

42 O’Callaghan C, Barry PW: The science of nebulised drug delivery. Thorax 1997;52(suppl 2):S31-S44.

$\checkmark 43$ Hess DR: Nebulizers: principles and performance. Respir Care 2000:45:609-622.

44 Boe J, Dennis JH, O’Driscoll BR, et al: European Respiratory Society Guidelines on the use of nebulizers. Eur Respir J 2001;18:228242.

-45 Hess DR, Fisher D, Williams P, Pooler S, Kacmarek RM: Medication nebulizer performance. Effects of diluent volume, nebulizer flow, and nebulizer brand. Chest 1996;110: 498-505.
46 Skaria S, Smaldone GC: Omron NE U22: comparison between vibrating mesh and jet nebulizer. J Aerosol Med Pulm Drug Deliv 2010;23:173-180.

47 Dhand R: Nebulisers that use a vibrating mesh or plate with multiple apertures to generate aerosol. Respir Care 2002;47:14061416.

48 Coates AL, Green M, Leung K, et al: A comparison of amount and speed of deposition between the PARI LC STAR ${ }^{\circledR}$ jet nebulizer and an investigational eFlow ${ }^{\circledR}$ nebulizer. J Aerosol Med Pulm Drug Deliv 2011;24:157163.

49 Nikander K: Adaptive aerosol delivery: the principles. Eur Respir Rev 1999;7:385-387.

50 Denyer J: Adaptive aerosol delivery in practice. Eur Respir Rev 1997;7:388-389.

51 Van Dyke RE, Nikander K: Delivery of iloprost inhalation solution with the HaloLite, Prodose, and I-neb adaptive aerosol delivery systems: an in vitro study. Respir Care 2007; 52:184-190.

52 Rubin BK: Pediatric aerosol therapy: new devices and new drugs. Respir Care 2011;56: 1411-1421.

53 Brand P, Schulte M, Wencker M, et al: Lung deposition of inhaled alpha1-proteinase inhibitor in cystic fibrosis and alpha1-antitrypsin deficiency. Eur Respir J 2009;34:354-360.

54 Brand P, Beckmann H, Maas Enriquez M, et al: Peripheral deposition of alpha-1 protease inhibitor using commercial inhalation devices. Eur Respir J 2003;22:262-267.

55 Mainz JG, Canisius S, Scheuch G, Mullinger B, Nocker K, Hofmann T: An open-label randomized pilot trial to evaluate tolerability, safety and applicability of budesonide inhalation suspension (BIS) delivered via AKITA JET in children aged 3-11 years with mild to moderate asthma (abstract). Chapel Hill, Congress of the International Society for Aerosols in Medicine, 2013.

56 Hofmann T: Optimized steroid delivery in severe and pediatric asthma: improved compliance and efficacy (abstract). Chapel Hill, Congress of the International Society for Aerosols in Medicine, 2013.
57 Janssens HM, Overweel J: Specific targeting of inhaled steroids to small airways in children with problematic severe asthma using the AKITA: a case series (abstract). Chapel Hill, Congress of the International Society for Aerosols in Medicine, 2013.

58 Dalby R, Spallek M, Voshaar T: A review of the development of Respimat soft mist inhaler. Int J Pharm 2004;283:1-9.

59 Kassner F, Hodder R, Bateman ED: A review of ipratropium bromide/fenoterol hydrobromide (Berodual) delivered via Respimat soft mist inhaler in patients with asthma and chronic obstructive pulmonary disease. Drugs 2004;64:1671-1682.

60 Lavorini F, Magnan A, Dubus JC, et al: Effect of incorrect use of dry powder inhalers on management of patients with asthma and COPD. Respir Med 2008;102:593-604.

61 Press VG, Pincavage AT, Pappalardo AA: The Chicago Breathe Project: a regional approach to improving education on asthma inhalers for resident physicians and minority patients. J Natl Med Assoc 2010;102:548-555.

62 Giraud V, Roche N: Misuse of corticosteroid metered-dose inhaler is associated with decreased asthma stability. Eur Respir J 2002;19: 246-251.

63 Melani AS, Bonavia M, Cilenti V, Cinti C, Lodi M, Martucci P, Serra M, Scichilone N, Sestini P, Aliani M, Neri M: Gruppo Educazionale Associazione Italiana Pneumologi Ospedalieri. Inhaler mishandling remains common in real life and is associated with reduced disease control. Respir Med 2011;105: 930-938.

64 Levy ML, Hardwell A, McKnight E, Holmes J: Asthma patients' inability to use a pressurised metered-dose inhaler (pMDI) correctly correlates with poor asthma control as defined by the Global Initiative for Asthma (GINA) strategy: a retrospective analysis. Prim Care Respir J 2013;22:406-411.

65 Lavorini F, Levy ML, Corrigan C, Crompton G; ADMIT Working Group: The ADMIT series - issues in inhalation therapy. 6) Training tools for inhalation devices. Prim Care Respir J 2010;19:335-341.

66 Price D, Bosnic-Anticevich S, Briggs A, et al: Inhaler competence in asthma: common errors, barriers to use and recommended solutions. Respir Med 2013;107:37-46.

67 Fink JB, Rubin BK: Problems with inhaler use: a call for improved clinician and patient education. Respir Care 2005;50:1360-1374. 